\title{
PARTITIONER-REPRESENTABLE ALGEBRAS
}

\author{
R. FRANKIEWICZ AND P. ZBIERSKI
}

(Communicated by Thomas J. Jech)

\begin{abstract}
We give a simple proof of the theorem of Baumgartner and Weese on representability of Boolean algebras. We also show that the representability of $P\left(\omega_{1}\right)$ implies the existence of a relative $Q_{3}$-set.
\end{abstract}

In [B-W], Baumgartner and Weese introduced so called partition algebras: if $E$ is a maximal antichain in $P(\omega) /$ fin, then an element $a \in P(\omega) /$ fin is called a partitioner of $E$ if, for each $e \in E$ either $e \leq a$ or $e \cdot a=0$ and the subalgebra of all the partitioners factorized $\bmod [E]([E]$ denotes the ideal generated by the set $E)$ is called the partition algebra of $E$. Finite sums $e_{1}+\cdots+e_{n}$ of elements of $E$ are called trivial partitioners. Of course, the notion of a partitioner of an antichain is meaningful in an arbitrary Boolean algebra.

Baumgartner and Weese proved the following

THEOREM 1. Assuming $\mathrm{CH}$, every Boolean algebra $A$, of cardinality $\leq c=2^{\omega}$, is partitioner-representable i.e. it is isomorphic to the partition algebra of a maximal antichain $E$ in $P(\omega) /$ fin.

We present below a simple proof of this theorem.

Observe that it suffices to construct a Parovičenko extension $A^{*}$ of $A$ and a antichain $E$ in $A^{*}$ such that the following two conditions are satisfied:

(1) Each nonzero element $a \in A$ is a nontrivial partitioner of $E$ (nontrivial means that $e \leq a$ holds for infinitely many $e \in E$ ).

(2) Each element $b \in A^{*} \backslash A$ is either a nonpartitioner or is congruent $\bmod [E]$ to an element of $A$.

Indeed, because of Parovicenko characterisation (see e.g. $[\mathrm{C}-\mathrm{N}]$ ) $A^{*}$ is (up to isomorphism) the algebra $P(\omega) /$ fin, each partitioner $b \in A^{*}$ is congruent $\bmod [E]$ to a unique element $f(b)$ of $A$ and the map $f$ is then a homomorphism with kernel $[E]$. Note, that (1) and (2) imply that $E$ is in fact a maximal antichain.

We define $A^{*}$ and $E$ as unions of increasing chains

$$
A^{*}=\bigcup\left\{A_{\alpha}: \alpha<\omega_{1}\right\}, \quad E=\bigcup\left\{E_{\alpha}: \alpha<\omega_{1}\right\},
$$

where the $A_{\alpha}$ 's and $E_{\alpha}$ 's are defined inductively, so that (the inductive assumption): each nonzero $a \in A$ is a partitioner of $E_{\alpha}$ but $a \notin\left[E_{\alpha}\right]$. We begin with $A_{0}=A$, $E_{0}=\varnothing$ and take unions at limit stages. It remains to describe the successor step. We may assume, that at each stage $\alpha$ we have fixed an enumeration of:

(a) All the nonzero elements of $A_{\alpha}$.

Received by the editors May 10, 1987.

1980 Mathematics Subject Classification (1985 Revision). Primary 03E50; Secondary 03E05, $06 \mathrm{E} 05$ 
(b) All decreasing chains $b_{0}>b_{1}>\cdots$, in $A_{\alpha}$.

(c) All chains of the form $b_{0}>b_{1}>\cdots>a_{1}>a_{0}$, in $A_{\alpha}$, so that at a given stage $\alpha$ we have an object $x$ of type (a), (b) or (c) namely, the $K(\alpha)$ th term of the $L(\alpha)$ th enumeration (where $K, L$ denote the inverses of a fixed pairing function: $\left.\omega \times \omega_{1} \rightarrow \omega_{1}\right)$.

Now, given $\alpha$, if $x$ is as in (b) or (c) we put $E_{\alpha+1}=E_{\alpha}$ and extend $A_{\alpha}$ by adding a new element below the $b_{n}$ 's, in case (b) or in between the $b_{n}$ 's and $a_{n}$ 's, in case (c). To do this, identify $A_{\alpha}$ with the field $B\left(X_{\alpha}\right)$ of open-closed sets of the associated Stone space $X_{\alpha}$ and take $A_{\alpha+1}$ as the subfield of $P\left(X_{\alpha}\right)$ generated by $A_{\alpha}=B\left(X_{\alpha}\right)$ and the set $b=\bigcap\left\{b_{n}: n<\omega\right\}$.

If $x$ is as in (a) we distinguish two cases. First, suppose $x \in A$. We may assume, that there is an atom $a \leq x$, disjoint from $E_{\alpha}$, for otherwise we replace $A_{\alpha}=B\left(X_{\alpha}\right)$ by the field generated by $A_{\alpha}$ and $\{p\}$, where $p \in x \backslash \bigcup E_{\alpha}$ (note, that - by the inductive assumption - the family $\left\{x \backslash \bigcup s: s \in E_{\alpha}^{<\omega}\right\}$ is centered). Let $Y=\left(X_{\alpha} \backslash a\right)+Z$ (direct sum), where $Z$ is one-point compactification of $\omega$, and put $A_{\alpha+1}=B(Y)$.

Thus, $A_{\alpha} \subseteq A_{\alpha+1}$ since $X_{\alpha}$ is a continuous image of $Y$ and in $A_{\alpha+1}$ there are infinitely many new atoms $e_{0}, e_{1}, \ldots$ below $a \leq x$ and all disjoint from $E_{\alpha}$. Let $E_{\alpha+1}=E_{\alpha} \cup\left\{e_{n}: n<\omega\right\}$. Thus, each element of $A$ remains a partitioner not in $\left[E_{\alpha+1}\right]$ and $x$ becomes a nontrivial partitioner.

Finally, suppose $x \in A_{\alpha} \backslash A$ and $x$ is a partitioner noncongruent $\bmod \left[E_{\alpha}\right]$ to elements of $A$ (if $x \in A_{\alpha} \backslash A$ is not such, we add only a new atom under $x$ and take $\left.E_{\alpha+1}=E_{\alpha}\right)$. Then, the complement $-x$ has the same property and the noncongruency condition implies that the ideal $I \subseteq A$, generated by the set $\{a \in$ $A: a \cdot x \in\left[E_{\alpha}\right]$ or $\left.a \cdot(-x) \in\left[E_{\alpha}\right]\right\}$ is proper. If $p$ is an ultrafilter in $A$ extending $-I=\{-a: a \in I\}$, then $\bigcap p \backslash \bigcup E_{\alpha}$ intersects both $x$ and $-x$, in $X_{\alpha}$. Hence, we can extend $A_{\alpha}$ by adding two new atoms: $e_{0} \leq x$ and $e_{1} \leq-x$, both disjoint from $E_{\alpha}$ and such that for each $a \in A$ either $e_{0}, e_{1} \leq a$ or $e_{0}, e_{1} \leq-a$. Defining $E_{\alpha+1}$ as $E_{\alpha} \cup\left\{e_{0}+e_{1}\right\}$ we see that the inductive assumption holds for $E_{\alpha+1}$ and $x$ becomes now a nonpartitioner. Q.E.D.

REMARK. As the referee pointed out, the Theorem above can be generalized for higher cardinalities as follows: Let $\kappa$ be an infinite cardinal. For every Boolean algebra $A$, of cardinality $\leq 2^{\kappa}$ there is an extension $A^{*}$ of cardinality $2^{\kappa}$ and a maximal antichain $E \subseteq A^{*}$ such that $A^{*}$ satisfies the condition $H\left(\kappa^{+}\right)[\mathrm{C}-\mathbf{N}$, p. 119] and $A$ is isomorphic to the partition algebra of $E$.

We prove now, that - consistently - there is an algebra of cardinality $c$, which is not partitioner-representable. Recall that an uncountable subset $X$ of Cantor space is said to be a $Q_{\alpha}$-set, if each subset $Y \subseteq X$ is Borel, relatively in $X$, of order at most $\alpha$. Miller, in $[\mathbf{M}]$, has constructed a model of ZFC in which there are no $Q_{\alpha}$-sets, for all $\alpha<\omega_{1}$ and $2^{\omega}=2^{\omega_{1}}=\omega_{2}$.

Hence, it suffices to prove the following

THEOREM 2. If the algebra $P\left(\omega_{1}\right)$ is partitioner-representable, then there is a $Q_{3}$-set.

ProOF. Let $A=P\left(\omega_{1}\right)$ (or, more generally, let $A$ be complete, atomic). By assumption, there is maximal antichain $E \subseteq P(\omega)$ /fin and an isomorphism $f$ from $A$ onto the partition algebra of $E$. For each nonzero $a \in A$ we take a set $S(a) \subseteq \omega$ 
such that

$$
f(a)=S(a) / \text { fin } /[E]
$$

Let us denote

$$
E(a)=\{e \in E: e \leq S(a) / \text { fin }\} .
$$

Thus, for $a>0, E(a)$ is infinite and

$$
\begin{aligned}
a & \leq b \text { implies } E(a) \backslash E(b) \text { is finite, } \\
a \circ b & =0 \text { implies } E(a) \cap E(b) \text { is finite. }
\end{aligned}
$$

Let $e_{n}^{a}=E_{n}^{a} /$ fin, for $a>0$ and $n<\omega$, be distinct elements from $E(a)$. The Cantor space $C$ will be represented as $C=\left(2^{\omega}\right)^{\omega}$. To each $a$ we assign an element $x^{a}=\left\langle x_{n}^{a}: n\langle\omega\rangle\right.$ of $C$ defined as follows: $x_{n}^{a}$ is $\chi_{E_{n}^{a}}$, the characteristic function of the set $E_{n}^{a}$. By $(*)$ the set

$$
X=\left\{x^{a}: a \text { is an atom of } A\right\}
$$

has cardinality $\omega_{1}$. To see that $X$ is a $Q_{3}$-set let

$$
K_{n}(a)=\left\{x \in C: x_{n} \leq \chi_{S(a)}\right\}
$$

(where $x \leq y$, for $x, y \in 2^{\omega}$, means that the set $\{n: x(n)>y(n)\}$ is finite) and let

$$
q(a)=\bigcap_{n<\omega} \bigcup_{i>n} K_{i}(a)
$$

Thus, for each $a, q(a)$ is an $F_{\sigma \delta}$, in $C$ and $x^{a} \in q(b)$ iff for infinitely many $n$ we have $E_{n}^{a} \subseteq S(b)$ (i.e. $E_{n}^{a} \backslash S(b)$ is finite). From (*) we obtain immediately

$$
\begin{array}{rll}
a \leq b & \text { implies } & x^{a} \in q(b), \\
a \cdot b=0 & \text { implies } & x^{a} \notin q(b) .
\end{array}
$$

Using the above formulas we infer at once, that for an arbitrary $Y \subseteq X$ :

$$
Y=q\left(b_{Y}\right) \cap X
$$

where $b_{Y}=\bigcup\left\{a: x^{a} \in Y\right\}$. Q.E.D.

\section{REFERENCES}

[B-W] J. E. Baumgartner and M. Weese, Partition algebras for almost-disjoint sets, Trans. Amer. Math. Soc. 274 (1982), 619-630.

[C-N] W. Comfort and S. Negrepontis, The theory of ultrafilters, Springer-Verlag, Berlin and New York, 1974.

[M] A. Miller, On the length of Borel hierarchies, Ann. Math. Logic 16 (1979), 233-267.

Mathematical institute, Polish Academy of Sciences, Sniadeckich 8, WaRSZAWA, POLAND

University of WarsaW, Department of Mathematics, PKin IXP., WarszaWa, POLAND 